\title{
T-Wave Morphology Changes as Surrogate for Blood Potassium Concentration in Hemodialysis Patients
}

\author{
Flavio Palmieri ${ }^{1,2,3}$, Pedro Gomis ${ }^{1,2}$, Dina Ferreira ${ }^{3}$, José Esteban Ruiz ${ }^{4}$, Beatriz Bergasa ${ }^{4}$, Alba \\ Martín-Yebra $^{5}$, Hassaan A. Bukhari ${ }^{2,6}$, Esther Pueyo ${ }^{2,6}$, Juan Pablo Martínez ${ }^{2,6}$, Julia Ramírez ${ }^{7}$, \\ Pablo Laguna ${ }^{2,6}$ \\ ${ }^{1}$ Centre de Recerca en Enginyeria Biomèdica, Universitat Politècnica de Catalunya, Barcelona, Spain \\ ${ }^{2}$ CIBER en Bioingeniera, Biomateriales y Nanomedicina (CIBER-BBN), Zaragoza, Spain \\ ${ }^{3}$ Laboratorios Rubio, Castellbisbal, Barcelona, Spain \\ ${ }^{4}$ Nephrology Department, Hospital Clínico Universitario Lozano Blesa, Zaragoza, Spain \\ ${ }^{5}$ Department of Biomedical Engineering, Lund University, Lund, Sweden \\ ${ }^{6}$ BSICoS Group, I3A, IIS Aragón, Universidad de Zaragoza, Zaragoza, Spain \\ ${ }^{7}$ William Harvey Research Institute, Queen Mary University of London, London, United Kingdom
}

\begin{abstract}
End-stage renal disease (ESRD) patients undergoing hemodialysis (HD) are at high risk of arrhythmias and sudden cardiac death as a result of blood potassium concentration $\left(\left[\mathrm{K}^{+}\right]\right)$changes. The aim of this study is to investigate if $d_{w}$, a time-warping-based electrocardiogram (ECG) biomarker of T-wave morphology changes, reflects $\left[\mathrm{K}^{+}\right]$evolution in $\mathrm{HD}$ patients, facilitating noninvasive $\left[\mathrm{K}^{+}\right]$monitoring and avoiding in-hospital blood tests analysis. 48-hour ECGs and a set of hourly-collected blood samples from 12 ESRD patients were acquired and analyzed. $d_{w}$ was calculated between a reference T-wave, measured at the end of the HD session, and the T-waves corresponding to each hour along the whole HD session, when $\left[\mathrm{K}^{+}\right]$was measured from blood samples. The values of $d_{w}$ correlated with the relative variations in $\left[\mathrm{K}^{+}\right]$ with respect to the reference value (end of $H D, \Delta\left[K^{+}\right]$), with a median (interquartile) correlation coefficient of 0.90 (0.30), evidencing a strong relation between them. Our findings support the use of $d_{w}$ as a surrogate of $\Delta\left[K^{+}\right]$, suggesting a potential use of $d_{w}$ for non-invasive hyperkalemia monitoring both in hospital and ambulatory settings.
\end{abstract}

\section{Introduction}

Variations in blood potassium concentration $\left(\Delta\left[K^{+}\right]\right)$ can alter the spatio-temporal ventricular repolarization sequence, resulting in abnormal dispersion of ventricular repolarization and thus increasing the susceptibility for malignant arrhythmias [1,2].
End-stage renal disease (ESRD) patients undergoing hemodialysis (HD) have a high prevalence of arrhythmias [3] and high cardiovascular (CV) mortality rates [1] due to fluctuations of $\left[\mathrm{K}^{+}\right]$out of normal ranges $(3.5 \mathrm{mmol} / \mathrm{L}$ $\leq \mathrm{K}^{+} \leq 5 \mathrm{mmol} / \mathrm{L}$ ) [2]. Currently, all the routine tests to assess $\left[\mathrm{K}^{+}\right]$require blood analyses, an invasive and costly procedure that limits a continuous monitoring [4]. A non-invasive ambulatory index able to quantify changes in $\left[\mathrm{K}^{+}\right]$levels would be an important advance for both inhospital and out-hospital continuous monitoring.

Abnormalities in ventricular repolarization are reflected on the electrocardiogram (ECG) signal, in particular, on the T-wave morphology [5]. Different ECG biomarkers have been previously reported to be correlated with $\left[\mathrm{K}^{+}\right]$ levels, including the T-wave slope-to-amplitude ratio [6] or the T-wave right slope [7]. However, these biomarkers rely on specific local features of some parts the T-wave, but neglect the information contained in the whole T-wave morphology. In this study, we investigated the ability of $d_{w}$, a time-warping-based ECG marker of T-wave morphology changes, with known association with ventricular repolarization dispersion [8], to monitor changes in $\left[\mathrm{K}^{+}\right]$levels in ESRD patients undergoing HD.

\section{Study Population and Protocol}

A standard, 12-lead 48-hour ECG Holter recording (H12+, Mortara Instruments, Milwaukee, WI, USA, sampling frequency $1000 \mathrm{~Hz}$, amplitude resolution $3.75 \mu \mathrm{V}$ ) and $\left[\mathrm{K}^{+}\right]$concentrations from blood samples were collected from 12 ESRD patients undergoing HD at Hospital Clínico Universitario Lozano Blesa (Zaragoza, Spain). 
Table 1 shows the population characteristics. The study protocol was approved by the ethical committee and all patients signed informed consent. The ECG recording started 5 minutes before the HD onset and lasted for 48 hours (Figure 1 blue line). Simultaneously, six blood samples were acquired and analyzed during the HD session: the first one at the HD onset and the next three every hour during the HD session (Figure 1, K1 to K4 in red). The $5^{\text {th }}$ blood sample was collected at the end of the HD (minute $215^{t h}$ or $245^{t h}$, depending on the HD session duration) while the $6^{\text {th }}$ blood sample was taken after 48 hours, immediately before the next HD session.

Table 1. Characteristics of the study population.

\begin{tabular}{lr}
\hline \hline Characteristics & All (n=12) \\
\hline Age (years) & $70(11)$ \\
Gender (male) & $7(58 \%)$ \\
Anti-arrhythmic drugs (yes) & $6(50 \%)$ \\
HD session duration & \\
240 min & $10(83 \%)$ \\
210 min & $2(17 \%)$ \\
Kidney disease etiology & \\
Diabetes mellitus & $7(58 \%)$ \\
Interstitial nephritis & $1(9 \%)$ \\
Tuberous sclerosis & $1(9 \%)$ \\
Other & $3(25 \%)$ \\
HD liquid Composition & $12(100 \%)$ \\
Potassium (1.5 mmol/L) & $8(67 \%)$ \\
Calcium (3 mmol/L) & \\
\hline \hline * Values are expressed as number (\%) for categorical variables \\
and median (interquartile range) for continuous variables
\end{tabular}

\begin{tabular}{|c|c|c|c|c|c|c|}
\hline \multicolumn{2}{|c|}{$\longleftarrow$} & \multicolumn{3}{|c|}{ ECG acquisition } & \multirow{2}{*}{ Post HD } & \multirow[b]{2}{*}{$\overrightarrow{\mathrm{K} 6}$} \\
\hline$\overleftarrow{K} 1$ & $\mathrm{~K} 2$ & $\begin{array}{l}\mathbf{H D} \\
\mathrm{K} 3\end{array}$ & K4 & K5 & & \\
\hline 5 & 65 & 125 & & $\begin{array}{l}245 \\
\text { (min) }\end{array}$ & & 2880 \\
\hline
\end{tabular}

Figure 1. Diagram of the study protocol. K1 to K6 are the time points (in minutes) for blood sample extraction. K5 was taken at the end of the HD (minute $215^{\text {th }}$ or $245^{\text {th }}$, depending on the HD duration).

\section{Methods}

\section{1. $\quad$ ECG pre-processing}

Initial ECG pre-processing included low-pass filtering with cut-off frequency at $40 \mathrm{~Hz}$ to remove muscular and powerline noise, and $0.5 \mathrm{~Hz}$ cut-off high-pass filtering to attenuate baseline wander. QRS complexes were detected and delineated using a wavelet-based single-lead delineation method applied to each of the 12 leads [9]. Principal component analysis was applied to the 8 independent leads. Principal components (PCs) were obtained from the auto-correlation matrix of T-waves computed in a stable ECG segment at the end of the HD session. Then, the whole ECG recording was projected into the direction given by the PC1. This way, the energy of the $\mathrm{T}$ wave in this component was maximized, allowing better morphological characterization [10]. The T-waves were further delineated by applying the same delineation method [9] to PC1.

\subsection{T-wave morphology changes quantifi- cation by time-warping}

The T-waves from PC1 were selected from a 2-min window centered on the $5^{\text {th }}$ minute of each available hour (Figure 2 (a)), as this is when the blood samples were collected during HD. The window was narrow enough to allow the assumption of stability in heart rate and $\left[\mathrm{K}^{+}\right]$levels. A mean warped T-wave (MWTW) was computed from the T-waves in each 2-min window [8]. For this, all the Twaves within each window were aligned with respect to their gravity center and used to compute an initial average warped T-wave [8]. Only those T-waves highly correlated (Spearman's correlation coefficient $>0.98$ ) with this initial average warped T-wave were used to recalculate the MWTW, which is representative of the T-wave morphology in that particular time window. The MWTW at the end of the HD treatment was taken as the reference, given that it is when the patient (i) has recovered the normal $\left[\mathrm{K}^{+}\right]$ level and (ii) is discharged from hospital, consequently being an appropriate reference for out-hospital ambulatory monitoring until next HD session.

Let's consider each hourly MWTW, $\boldsymbol{f}^{s}\left(\boldsymbol{t}^{s}\right)=$ $\left[f^{s}\left(t^{s}(1)\right), \ldots, f^{s}\left(t^{s}\left(N_{s}\right)\right)\right]^{\top}$, and the reference MWTW, $\boldsymbol{f}^{r}\left(\boldsymbol{t}^{r}\right)=\left[f^{r}\left(t^{r}(1)\right), \ldots, f^{r}\left(t^{r}\left(N_{r}\right)\right)\right]^{\top}$, respectively, from the red and blue squares in Figure 2 (a); where $\boldsymbol{t}^{r}=$ $\left[t^{r}(1), \ldots, t^{r}\left(N_{r}\right)\right]^{\top}$ and $\boldsymbol{t}^{s}=\left[t^{s}(1), \ldots, t^{s}\left(N_{s}\right)\right]^{\top}$ and $N_{r}$ and $N_{s}$ are the total duration of $\boldsymbol{t}^{r}$ and $\boldsymbol{t}^{s}$. As an example, let's assume $\boldsymbol{f}^{s}\left(\boldsymbol{t}^{s}\right)$ as the MWTW from the $1^{\text {st }}$ hour (red filled square in Figure 2 (a)), then $\boldsymbol{f}^{s}\left(\boldsymbol{t}^{s}\right)=\boldsymbol{f}^{1}\left(\boldsymbol{t}^{1}\right)$, $\boldsymbol{t}^{s}=\boldsymbol{t}^{1}$ and $N_{s}=N_{1}$. Figure 2 (b) shows the two $\boldsymbol{t}^{r}$ and $\boldsymbol{t}^{1}$ MWTWs before the time-warping. Let $\gamma\left(\boldsymbol{t}^{r}\right)$ be the warping function that relates $\boldsymbol{t}^{r}$ and $\boldsymbol{t}^{1}$, such that the composition $\left[\boldsymbol{f}^{1} \circ \gamma\right]\left(\boldsymbol{t}^{r}\right)=\boldsymbol{f}^{1}\left(\gamma\left(\boldsymbol{t}^{r}\right)\right)$ denotes the reparameterization or time domain warping of $f^{1}\left(t^{1}\right)$ using $\gamma\left(\boldsymbol{t}^{r}\right)$. The square-root slope function (SRSF) was proposed [11] to find the optimal warping function by warping the SRSFs of the original T-waves. This transformation is 
defined as:

$$
\boldsymbol{q}_{f}(\boldsymbol{t})=\operatorname{sign}(\dot{\boldsymbol{f}}(\boldsymbol{t})) \sqrt{|\dot{\boldsymbol{f}}(\boldsymbol{t})|}
$$

The optimal warping function is the one that minimizes the amplitude difference between the SRSF of $\boldsymbol{f}^{r}\left(\boldsymbol{t}^{r}\right)$ and $\boldsymbol{f}^{1}\left(\gamma\left(\boldsymbol{t}^{r}\right)\right)[11]$ :

$$
\begin{aligned}
\gamma^{*}\left(\boldsymbol{t}^{r}\right) & =\underset{\gamma\left(\boldsymbol{t}^{r}\right)}{\arg \min }\left(\left\|\boldsymbol{q}_{f^{r}}\left(\boldsymbol{t}^{r}\right)-\boldsymbol{q}_{\left[f^{1} \circ \gamma\right]}\left(\boldsymbol{t}^{r}\right)\right\|\right) \\
& =\underset{\gamma\left(\boldsymbol{t}^{r}\right)}{\arg \min }\left(\left\|\boldsymbol{q}_{f^{r}}\left(\boldsymbol{t}^{r}\right)-\boldsymbol{q}_{f^{1}}\left(\gamma\left(\boldsymbol{t}^{r}\right)\right) \sqrt{\dot{\gamma}\left(\boldsymbol{t}^{r}\right)}\right\|\right)
\end{aligned}
$$

The dynamic programming algorithm was used to obtain the solution of this optimization problem [12]. The optimal warping function, $\gamma^{*}\left(\boldsymbol{t}^{r}\right)$, that optimally warps $\boldsymbol{f}^{r}\left(\boldsymbol{t}^{r}\right)$ and $\boldsymbol{f}^{1}\left(\boldsymbol{t}^{1}\right)$ is shown in Figure 2 (c). The warped T-wave, $\boldsymbol{f}^{1}\left(\gamma^{*}\left(\boldsymbol{t}^{r}\right)\right)$ is shown in Figure $2(\mathrm{~d})$, together with the reference T-wave, $\boldsymbol{f}^{r}\left(\boldsymbol{t}^{r}\right)$.

The index $d_{w}$ (Figure 2 (c)) quantifies the level of warping needed to optimally align any two T-waves as the average of the absolute difference value between $\gamma^{*}\left(\boldsymbol{t}^{r}\right)$ and $\boldsymbol{t}^{r}$ :

$$
d_{w, 1}=\frac{1}{N_{r}} \sum_{n=1}^{N_{r}}\left|\gamma^{*}\left(t^{r}(n)\right)-t^{r}(n)\right|
$$

This was repeated for each hour.

The relative variations in $\left[\mathrm{K}^{+}\right]$with respect to the reference values (end of treatment), during the HD session were computed as:

$$
\Delta\left[K^{+}\right]=\left(\left[K^{+}\right]_{s}-\left[K^{+}\right]_{r}\right)
$$

\section{4. $\quad$ Results}

None of the 12 ECG recordings lasted 48h, mainly due to electrode detachment or early battery exhaustion. This limited the association between $d_{w}$ and the last $\left[\mathrm{K}^{+}\right]$sample (after 48h). For that reason, Spearman's correlation coefficients were computed considering only the first five values of $\Delta\left[\mathrm{K}^{+}\right]$and $d_{w}$ (those obtained during the HD session). The median (interquartile range) value computed over all the intra-subject Spearman's correlation coefficients was found to be $0.90(0.30)$, indicating a significant correlation between $\Delta\left[\mathrm{K}^{+}\right]$and $d_{w}$. Figure 3 shows the distribution of $d_{w}$ and $\Delta\left[K^{+}\right]$during the HD session.

\section{Discussion}

Evaluation of $\left[\mathrm{K}^{+}\right]$levels is usually performed by invasive procedures, based on blood samples that require further laboratory analyses. However, it is known that the ECG can reflect changes correlated to $\left[\mathrm{K}^{+}\right]$variations. ESRD patients undergoing HD present important $\left[\mathrm{K}^{+}\right]$
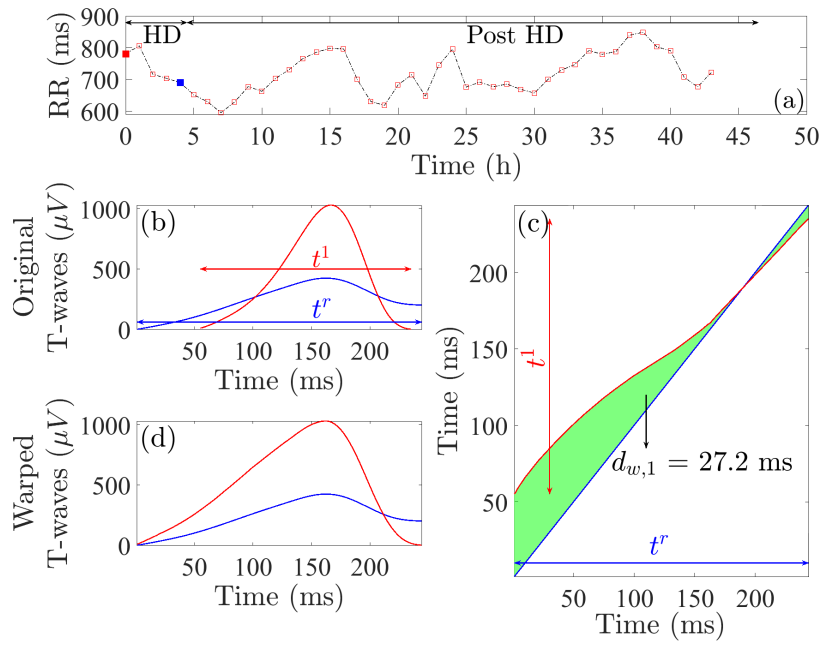

Figure 2. Illustration of $d_{w}$ computation for a subject undergoing a 240-min HD session. The average RR interval is shown in panel (a) with squares depicting the measurement windows when $d_{w}$ was computed during the ECG acquisition. The filled red square is the measurement at the beginning of HD and the filled blue square is the reference time at the end of HD. In panel (b), the reference (in blue) and the MWTW computed for the first hour (in red) are shown. Panel (c) shows the warping function (red line) that optimally relates them and the green area is the total warping information, quantified by $d_{w}$. Panel (d) shows the final warped T-waves.

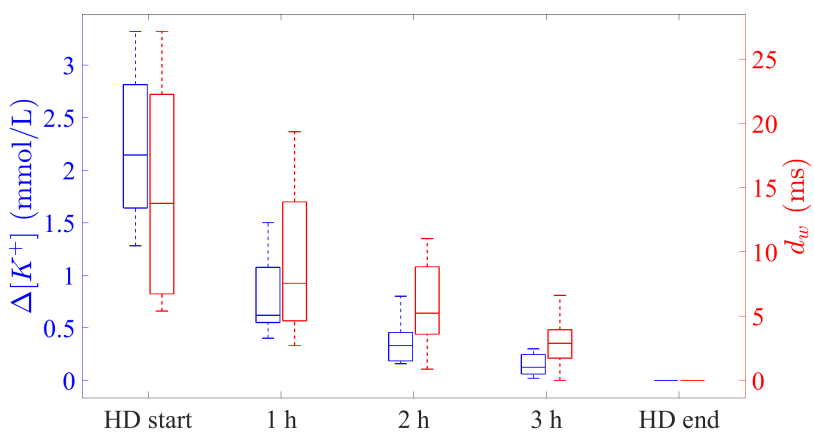

Figure 3. Distribution of $\Delta\left[\mathrm{K}^{+}\right]$(in blue, left verticalaxis) and $d_{w}$ (in red, right vertical-axis), computed at different time points from the beginning to the end of the HD session (K1 to K5 in Figure 1).

level fluctuations during the treatment, generating related ECG signal variations. Indeed, during the first hour of the HD procedure, $\left[\mathrm{K}^{+}\right]$quickly decreases. This acute fall in $\left[\mathrm{K}^{+}\right]$is followed by a more gradual decline over the next 2 hours. During the final hour, $\left[\mathrm{K}^{+}\right]$remains almost stable, indicating equilibrium between the rates of $\left[\mathrm{K}^{+}\right]$removal and the rate of re-equilibration from intracellular space. After HD treatment ends, a sub-acute rebound of $\left[\mathrm{K}^{+}\right]$oc- 
curs as continued mobilization of $\left[\mathrm{K}^{+}\right]$from intracellular to extracellular space occurs.

As explained in [7], action potential duration in endocardial, mid-myocardial and epicardial myocytes are differently affected by extracellular $\left[\mathrm{K}^{+}\right]$and this is reflected mostly on the ECG as T-wave changes. This makes the analysis of T-wave morphology changes a suitable $\left[\mathrm{K}^{+}\right]$ level sensor. In this work, we have shown how HD-induced electrolyte changes affect T-wave morphology. The intrasubject Spearman's correlation coefficients computed between $\Delta\left[\mathrm{K}^{+}\right]$and $d_{w}$ during the HD session are remarkable: the median value computed over all the enrolled participants is 0.90 and interquartile range 0.3 , supporting our initial hypothesis. Based on the evidences provided in this study, $d_{w}$ is suggested as a non-invasive index able to capture fluctuations in $\left[\mathrm{K}^{+}\right]$. Therefore, $d_{w}$ could be used to monitor patients susceptible to $\left[\mathrm{K}^{+}\right]$disorders. Its capacity for arrhythmic risk prediction could be assessed as well in those populations. The advantage of $d_{w}$ as a non-invasive automated surrogate for $\left[\mathrm{K}^{+}\right]$trend monitoring is that it would reduce the costs of $\left[\mathrm{K}^{+}\right]$invasive assessment, currently requiring blood test analyses, being especially suitable for daily routine check outside hospital environments.

There are several limitations in this study that should be acknowledged. First, the reduced number of patients enrolled in the study limited the statistical power of the results. Second, the lack of blood samples after the HD session limits the comparison between $d_{w}$ and $\Delta\left[\mathrm{K}^{+}\right]$ over that period.Nevertheless, the high correlation between $\Delta\left[\mathrm{K}^{+}\right]$and $d_{w}$ opens a new way to non-invasively monitor $\left[\mathrm{K}^{+}\right]$level changes.

Future studies should assess the correlation between $d_{w}$ and alterations in other electrolytes beyond $\left[\mathrm{K}^{+}\right]$, like magnesium or calcium. These variations can actually alter the ECG and it would be interesting to analyze their relationship with the induced T-wave changes. This could shed more light into ECG alterations in ESRD patients and the link to morbidity and mortality in these patients.

\section{Conclusions}

A novel ECG-based index, $d_{w}$, reflecting variation in ventricular repolarization from the ECG has been evaluated in ESRD patients undergoing HD and compared to changes in $\left[\mathrm{K}^{+}\right]$levels over time. The index $d_{w}$ is highly correlated with $\left[\mathrm{K}^{+}\right]$changes and could potentially be used as a non-invasive indicator of abnormal $\left[\mathrm{K}^{+}\right]$levels.

\section{Acknowledgments}

This work is funded by Products \& Technology S.L. (Castellbisbal, Barcelona, Spain), and by AGAUR, Generalitat de Catalunya (Spain). The work was also supported by project DPI2016-75458-R funded by MINECO and FEDER, by Gobierno de Aragón (Reference Group BSICoS T39-17R) cofunded by FEDER 2014-2020 "Building Europe from Aragón" and by European Research Council (ERC) through project ERC-2014-StG 638284. J. Ramírez acknowledges support from the Marie Sklodowska-Curie grant agreement No 786833.

\section{References}

[1] Ortiz A, et al. Epidemiology, contributors to, and clinical trials of mortality risk in chronic kidney failure. Lancet May 2014;383(9931):1831-1843.

[2] Secemsky E, et al. High prevalence of cardiac automonic dysfunction and T-wave alternans in dialysis patients. Heart Rhythm Apr 2011;8(4):592-598.

[3] Bignotto L, et al. Electrocardiographic findings in chronic hemodialysis patients. J Bras Nefrol Jul- Sep. 2012; 34(4):235-242.

[4] Hickner J, et al. Testing process errors and their harms and consequences reported from family medicine practices: a study of the american academy of family physicians national research network. Qual Saf Health Care Jun 2008; 17(3):194-200

[5] Webster A. Recognising signs of danger: ECG changes resulting from an abnormal serum potassium concentration. Emerg Med J Jan 2002;19(1):74-77.

[6] Corsi C, et al. Noninvasive quantification of blood potassium concentration from ECG in hemodialysis patients. Sci Rep Feb 2017;7(42492):doi: 10.1038/srep42492.

[7] Dillon J, et al. Noninvasive potassium determination using a mathematically processed ECG: Proof of concept for novel "blood-less, blood test". J Electrocardiol 2015;48(1):1218.

[8] Ramírez J, et al. Variability of ventricular repolarization dispersion quantified by time-warping the morphology of the T-wave. IEEE Trans Biomed Eng Jul 2017;64(7):16191630.

[9] Martínez JP, et al. A wavelet-based ECG delineator: Evaluation on standard databases. IEEE Trans Biomed Eng Apr 2004;4(51):570-581.

[10] Ramírez J, et al. Characterization of cardiac repolarization response to heart rate changes provoked by a tilt test. Comp in Cardiol 2012;39:673-679.

[11] Tucker JD, et al. Generative models for functional data using phase and amplitude separation. Comput Statist Data Anal May 2013;61:50-60.

[12] Bertsekas D. P. Dynamic Programming and Optimal Control, volume 1. Belmont, MA, USA: Athena Scientific, 1995.

Address for correspondence:

Flavio Palmieri; UPC CREB, Av Diagonal, 647, 08028 Barcelona (Spain). flavio.palmieri@upc.edu 